This is a postprint version of the following published document:

Monzón Baeza, V.; Sánchez-Fernández, M.; García Armada, A.; Royo, A. (2015). A Testbed for a LIFI System integrated in Streetlights. Networks and Communications (EuCNC), 2015 European Conference on, pp.517- 521. Available in http://dx.doi.org/10.1109/EuCNC.2015.7194129

C2015. IEEE. Personal use of this material is permitted. Permission from IEEE must be obtained for all other uses, in any current or future media, including reprinting/republishing this material for advertising or promotional purposes, creating new collective works, for resale or redistribution to servers or lists, or reuse of any copyrighted component of this work in other works. 


\title{
Testbed for a LiFi System integrated in Streetlights
}

\author{
Víctor Monzón Baeza ${ }^{1}$, Matilde Sánchez-Fernández ${ }^{1}$, Ana García Armada ${ }^{1}$ and Antonio Royo ${ }^{2}$ \\ ${ }^{1}$ Department of Signal Theory and Communications. Universidad Carlos III de Madrid, Spain \\ ${ }^{2}$ UVAX Concepts S.L., Parque Empresarial Táctica, Paterna Valencia, Spain \\ vmonzon@tsc.uc3m.es, mati@tsc.uc3m.es, agarcia@tsc.uc3m.es, a.royo@uvax-concepts.com
}

\begin{abstract}
In this paper, a functional $\mathrm{LiFi}$ real-time testbed implemented on FPGAs is presented. The setup evaluates the performance of our design in a downlink scenario where the transmitter is embedded on the streetlights and a mobile phone's camera is used as receiver, therefore achieving the goal of lighting and communicating simultaneously. To validate the design, simulations of the whole system are performed. where simulations of the channel between streetlight and mobile device show the scope of the reflection in an outdoor environment. The measurements are carried out to characterize the modulator, in particular the FPGA resources and latency due to the encoder blocks. In addition the feasibility of the communication function in presence of the ambient light is verified.
\end{abstract}

\section{INTRODUCTION}

The concept of Smart City has attracted a lot of interest in recent years; even though, it was coined at the beginning of 90s. [1]. A Smart City is known as a city committed to the environment, whose infrastructures are equipped with the most advanced and innovative technological solutions from the Information and Communications Technology area so as to satisfy the challenges of the cities in terms of energy sustainability (e.g., water, gas, electricity) to the social demand for vast real time information (about parking, public transport, weather, ATMs) and to the emergence of the Future Internetrelated technologies, such a wearable computing, mobile devices, cloud computing or the Internet of Things (IoT) [2], [3].

Many of technological solutions are based on the usage of WiFi by smartphones, which are increasing the continuous demand for higher data rates. In order to tackle this problem, network operators are forced to deploy new technologies like the future 5G [4]. As the RF spectrum is getting scarcer, the push for more bandwidth is driving wireless technologies in alternative spectrum bands into the networking field. Besides, supplying cities with wireless radio frequency networks is limited by the concern from the general public on the exposure to the electromagnetic fields, which is increasing nowadays. For instance, the 2010 Eurobarometer study [5] reveals that $70 \%$ of the European citizens believe that mobile phone base stations could have some negative effects on their health, which hinders the progress in these technologies.

In order to deal with the limited RF spectrum and social concern, Optical Wireless Communication (OWC) systems are getting an important attention both from the research community and the industry because of their numerous potential advantages, in particular, the usage of the freely available visible light spectrum in Visible Light Communication (VLC) systems [6].

Recently due to the widespread use of Light Emitting Diode (LEDs) in streetlights and the fact that these devices are the key element of optical systems, the design of technologies jointly performing the two functions -lighting and communicating- is made possible. In this case, the incorporation of VLC technology into LED bulb drive circuits provides the opportunity of applying the same visible light energy used for illumination and for communication too.

In the context of a home networking environment, Power Line Communication (PLC) is used to distribute high-speed data. PLC combined with VLC may benefit from the main infrastructure for backhaul and allow wireless connection between devices and the Internet. For this application, where LEDs are used as internet access points, VLC technology is known as LiFi (Light Fidelity) [7]. This term was coined firstly by Edinburgh University's Prof. Harald Haas in 2011 [8]. Many experts claim that LiFi represents the future of mobile internet thanks to its reduced costs and greater efficiency, becoming an alternative to $\mathrm{WiFi}$ as a basic technology for the IoT [2].

In this framework, our goal is to implement a LiFi system to validate its use in a scenario with streetlights. It focuses on a downlink system formed by the streetlight as a transmitter and a mobile phone camera as a receiver. The streetlights receive data via PLC and modulate the information to be sent by light pulses through the LED bulb. The modulator functionality is the one to be designed and tested within the work we present here. In turn, streetlights forward the data received by the optical link to the mobile user terminal which receives through a photodiode integrated in a camera. For the uplink, LiFi is integrated with existing technologies as Bluetooth, ZigBee or WiFi. Thanks to the infrastructure of streetlight that cities possess, they reach full connectivity and are able to interconnect other devices with each other such as traffic and vehicles lights or ATMs. The envisaged scenario is shown in Fig. 1.

In summary, the current work focuses on the following contributions:

- The design and implementation on Field Programmable Gate Array (FPGAs) of the modulator.

- Simulations of the channel between streetlight and mobile device.

- The definition of the testbed for testing LIFi in the streetlights. 


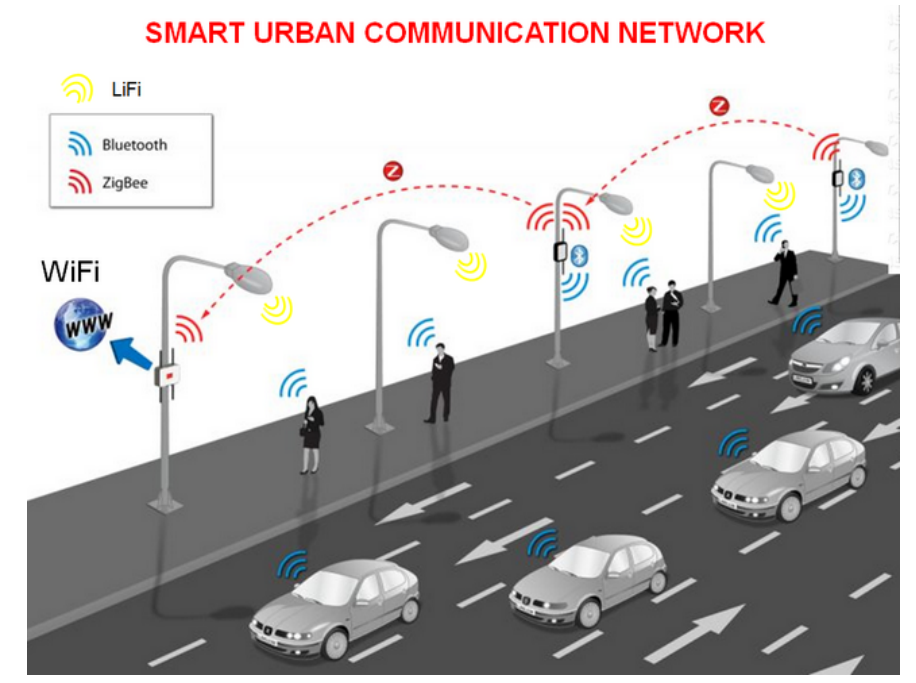

Fig. 1. LiFi Network in SmartCity.

The rest of the paper is organized as follows. In Section II LiFi standard [9] is briefly reviewed. In Section III a study and simulations for analyzing the design and the channel is presented. The system set up is analyzed in Section IV. Section V shows the results and experiments. Finally, Section VI presents the conclusions and ongoing work.

\section{LI-FI PHY LAYER OVERVIEW}

The physical (PHY) and the medium access control (MAC) layers for $\mathrm{LiFi}$ are specified in IEEE 802.15.7 standard [9]. Both the PHY and MAC layers are defined to achieve high data rates in audio and video transmission, while the illumination function is not affected.

As specified in the standard, there are three different types of PHY with various combinations of digital modulation and coding schemes [9]. The data rates are from $11.67 \mathrm{~kb} \mathrm{~kb} / \mathrm{s}$ to $266.6 \mathrm{~kb} / \mathrm{s}$ for the PHY I and from 1.25 Mb/s to $96 \mathrm{Mb} / \mathrm{s}$ for PHY II. For streetlights, the best option is the PHY I because it is optimized for outdoor scenarios. However, the PHY II is considered to be implemented too in this work.

The modulation schemes used are on-off-keying (OOK) and variable pulse position modulation (VPPM). Reed Solomon (RS) and Convolutional encoders are proposed by the standard to correct channel errors and improve the link reliability. On the other hand, to avoid burst errors, an interleaver is included. Manchester and 4B6B encoders are the two types of RLL encoders which are used for $200 \mathrm{kHz}$ and $400 \mathrm{kHz}$ clock rates, respectively, to balance the DC level of the output. The key combinations for PHY I-II are shown in Table I and II. For more details on the encoder parameters or modulation, the reader may check [9].

The standard does not include a specification for the buffers between blocks, but they are necessary to make data conversion between serial and parallel data flow, for example between RS and interleaver. Also, the implementation is not described in the standard thereby it is proposed in this paper and presented in Section IV.
TABLE I

PHY I OPERATION MODES

\begin{tabular}{|c|c|c|c|c|c|}
\hline \multirow{2}{*}{$\begin{array}{c}\text { Modula- } \\
\text { tion }\end{array}$} & \multirow{2}{*}{$\begin{array}{l}\text { RLL } \\
\text { code }\end{array}$} & \multirow{2}{*}{$\begin{array}{c}\text { Optical } \\
\text { clock rate }\end{array}$} & \multicolumn{2}{|c|}{ FEC } & \multirow[t]{2}{*}{ Date rate } \\
\hline & & & $\begin{array}{l}\text { Outer } \\
\text { code(RS) }\end{array}$ & $\begin{array}{l}\text { Inner } \\
\text { code }(\mathrm{CC})\end{array}$ & \\
\hline \multirow{5}{*}{ OOK } & \multirow{5}{*}{ Manchester } & \multirow{5}{*}{$200 \mathrm{KHz}$} & $(15,7)$ & $1 / 4$ & $11.67 \mathrm{~kb} / \mathrm{s}$ \\
\hline & & & $(15,11)$ & $1 / 3$ & $24.44 \mathrm{~kb} / \mathrm{s}$ \\
\hline & & & $(15,11)$ & $2 / 3$ & $48.89 \mathrm{~kb} / \mathrm{s}$ \\
\hline & & & $(15,11)$ & None & $73.3 \mathrm{~kb} / \mathrm{s}$ \\
\hline & & & None & None & $100 \mathrm{~kb} / \mathrm{s}$ \\
\hline \multirow{4}{*}{ VPPM } & \multirow{4}{*}{ 4B6B } & \multirow{4}{*}{$400 \mathrm{KHz}$} & $(15,2)$ & $1 / 4$ & $35.56 \mathrm{~kb} / \mathrm{s}$ \\
\hline & & & $(15,4)$ & None & $71.11 \mathrm{~kb} / \mathrm{s}$ \\
\hline & & & $(15,7)$ & None & $124.4 \mathrm{~kb} / \mathrm{s}$ \\
\hline & & & None & None & $266.6 \mathrm{~kb} / \mathrm{s}$ \\
\hline
\end{tabular}

TABLE II

PHY II OPERATION MODES

\begin{tabular}{|c|c|c|c|c|}
\hline $\begin{array}{c}\text { Modula- } \\
\text { tion }\end{array}$ & $\begin{array}{l}\text { RLL } \\
\text { code }\end{array}$ & $\begin{array}{c}\text { Optical } \\
\text { clock rate }\end{array}$ & FEC & Date rate \\
\hline \multirow{5}{*}{ VPPM } & \multirow{5}{*}{$4 \mathrm{~B} 6 \mathrm{~B}$} & \multirow{2}{*}{$3.75 \mathrm{MHz}$} & $\operatorname{RS}(64,32)$ & $1.25 \mathrm{Mkb} / \mathrm{s}$ \\
\hline & & & $\mathrm{RS}(160,128)$ & $2 \mathrm{Mb} / \mathrm{s}$ \\
\hline & & \multirow{3}{*}{$7.5 \mathrm{MHz}$} & $\mathrm{RS}(64,32)$ & $2.5 \mathrm{Mb} / \mathrm{s}$ \\
\hline & & & $\mathrm{RS}(160,128)$ & $4 \mathrm{Mb} / \mathrm{s}$ \\
\hline & & & None & $5 \mathrm{Mb} / \mathrm{s}$ \\
\hline \multirow{9}{*}{ OOK } & \multirow{9}{*}{ 8B10B } & \multirow{2}{*}{$15 \mathrm{MHz}$} & $\operatorname{RS}(64,32)$ & $6 \mathrm{Mb} / \mathrm{s}$ \\
\hline & & & $\mathrm{RS}(160,128)$ & $9.6 \mathrm{Mb} / \mathrm{s}$ \\
\hline & & \multirow{2}{*}{$30 \mathrm{MHz}$} & $\mathrm{RS}(64,32)$ & $12 \mathrm{Mb} / \mathrm{s}$ \\
\hline & & & $\mathrm{RS}(160,128)$ & $19.2 \mathrm{Mb} / \mathrm{s}$ \\
\hline & & \multirow{2}{*}{$60 \mathrm{MHz}$} & $\mathrm{RS}(64,32)$ & $24 \mathrm{Mb} / \mathrm{s}$ \\
\hline & & & $\mathrm{RS}(160,128)$ & $38.4 \mathrm{Mb} / \mathrm{s}$ \\
\hline & & \multirow{3}{*}{$120 \mathrm{MHz}$} & $\mathrm{RS}(64,32)$ & $48 \mathrm{Mb} / \mathrm{s}$ \\
\hline & & & $\mathrm{RS}(160,128)$ & $76.8 \mathrm{Mb} / \mathrm{s}$ \\
\hline & & & None & $96 \mathrm{Mb} / \mathrm{s}$ \\
\hline
\end{tabular}

\section{System Design AND Simulation}

The development of this project started with simulations in MATLAB of a reference LiFi modulator and demodulator according to the specifications of [9] for PHY I-II operating modes. The study of the model in MATLAB serves as a benchmark for subsequent implementation in FPGA for streetlights.

The simulation is performed in two parts that are described below.

\section{A. Simulation of LiFi PHY layer}

First, the system viability is tested. To do this, each of the blocks that make up the $\mathrm{LiFi}$ transmitter and receiver are programmed in MATLAB: RS and convolutional encoder, interleaver, Manchester and 4B6B encoding; while in the receptor, the Viterbi algorithm is performed first and then blocks transmission coders are undone. In Fig. 2 it is shown the block diagram.

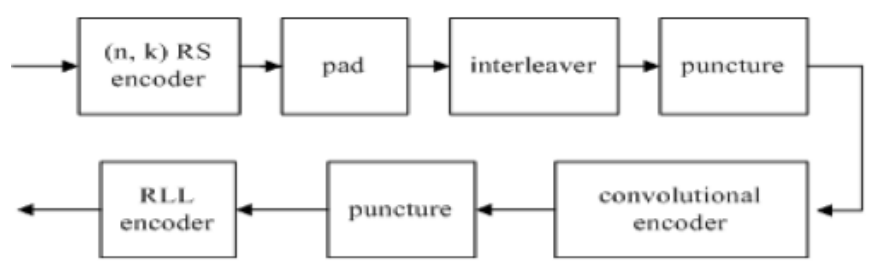

Fig. 2. Reference modulator diagram for PHY I. 
The results according the bit error rate (BER) are shown in the Fig. 3 for AWGN (Additive White Gaussian Noise). As shown, the operating mode, that has the lowest data rate according to the Table I, presents the best performance because of the high protection. $\mathrm{SNR}=10 \mathrm{~dB}$ is chosen to illustrate the effect on an image transmission using our simulated $\mathrm{LiFi}$ system. In Fig. 4 the quality of the image clearly shows the effect of coding. Also, if a SNR $=15 \mathrm{~dB}$ is chosen, a level of BER and image quality are acceptable for all modes of operation.

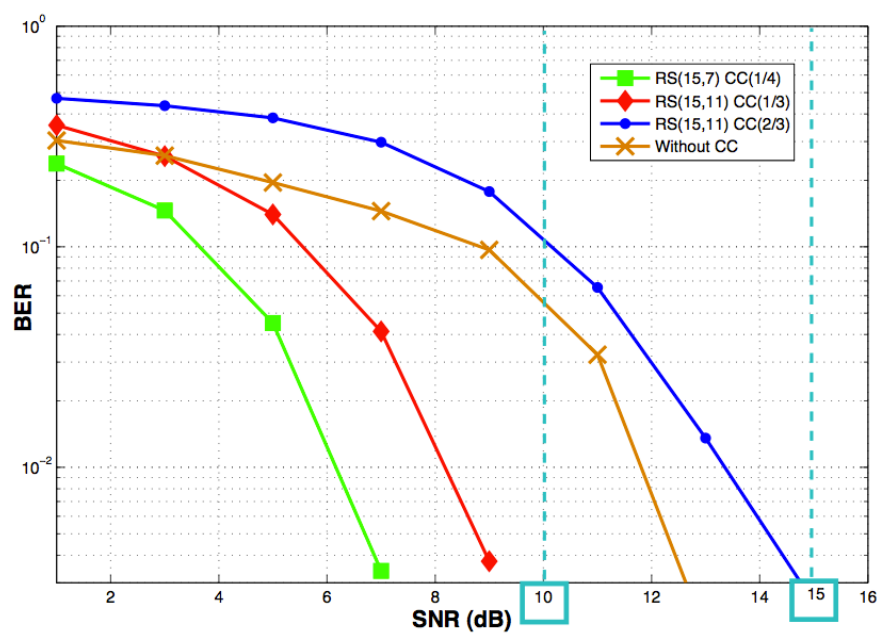

Fig. 3. Performance.
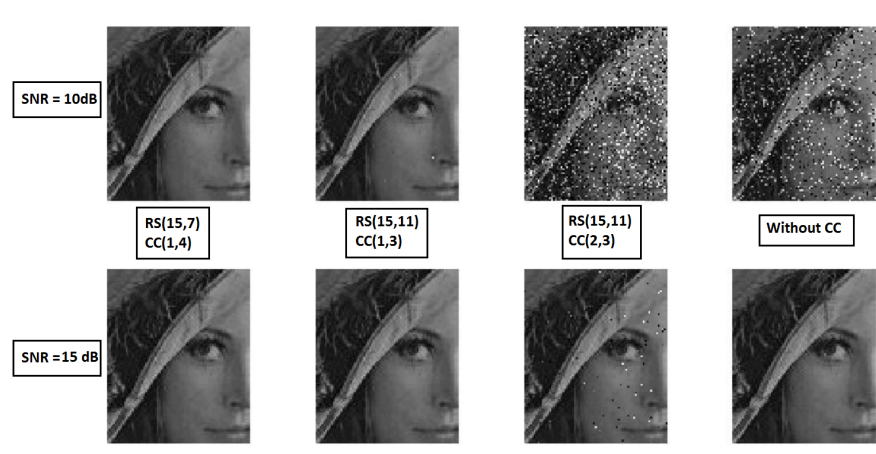

Fig. 4. Image transmission using LiFi system.

\section{B. Channel model}

The second study phase was dedicated to understand the characteristics of the channel, as it is important for a proper design and implementation. Characterization is performed by the channel impulse response, which is then used to analyze and combat the effects of channel distortions. In this scenario the channel is defined between the streetlight and phone's camera, so it is an atmospheric outdoor channel. Two types of configurations may be considered according to (1) the degree of directionality of transmitter and receiver and (2) the existence of the line of sight (LOS) path between the transmitter and the receiver.
The link is considered to have LOS between the streetlight and mobile. It does not suffer the effects of multipath fading, but there may be scattering effects due to reflections from objects. These parameters are simulated in this work. According to [10], since the distance between transmitter and receiver is small, the channel response $h(t ; S, R)$, where $S$ and $R$ are the position of the transmitter and receiver respectively, is approximately equal to that in indoor applications so defined as

$$
h(t ; S, R)=\sum_{k=0}^{\infty} h^{(k)}(t ; S, R)
$$

where $k$ denotes the reflection order. For $k=0, h^{(0)}(t ; S, R)$, the expression is

$$
h^{(0)}(t ; S, R) \approx \frac{n+1}{2 \pi} \cos ^{n}(\phi) d \Omega \Pi(\theta / F O V) \delta(t-d / c)
$$

Here, $n$ is the mode number which specifies the directionality of the source. $(\phi, \theta)$ define the source radiation lobe. Field of View $(F O V)$ is that solid angle through which a receiver is sensitive to detect light. This may be used to reduce unwanted reflections or noise. $d \Omega$ is the receiver solid angle, $d \Omega \approx$ $\cos (\theta) A_{R} / d^{2}$, where $A_{R}$ is detector area and $d$ the distance between the source and receiver. The term $\delta$ represents the delay of the beam. To calculate the $k^{\text {th }}$ reflection

$$
h^{(k)}(t ; S, R)=\int_{S} h^{(0)}\left(t ; S,\left\{r, \hat{n}, \pi / 2, d r^{2}\right\}\right) \otimes h^{k-1}(t ;\{r, \hat{n}, 1\}, R)
$$

First, $h(t ; S, R)$ is simulated, using the values of Table III,

\section{TABLE III}

\begin{tabular}{|c|c|c|c|}
\hline \multicolumn{2}{|c|}{ Transmitter } & \multicolumn{2}{c|}{ Receiver } \\
\hline \hline Angle of emission & $\phi=-90^{\circ} \theta=0^{\circ}$ & Detector Area $\left(A_{R}\right)$ & $1 \mathrm{~cm}^{2}$ \\
\hline LED Power & $1 \mathrm{~W}$ & FOV & 85 \\
\hline S( x,y,x) & $(2.5,2.5,3)$ & $\mathrm{R}(\mathrm{x}, \mathrm{y}, \mathrm{x})$ & $(1.5,1.5,0)$ \\
\hline Reflectivity & 0.8 & Number of reflection & 3 \\
\hline mode number $n$ & 1 & $\mathrm{~d}$ & $1 \mathrm{~m}$ \\
\hline
\end{tabular}

provided by supplier, to determine the effect of the reflections on reception. Fig. 5 shows the power delay profile (PDP) in this scenario. The reflections do not create any problems for detection because the direct beam is much stronger than others.

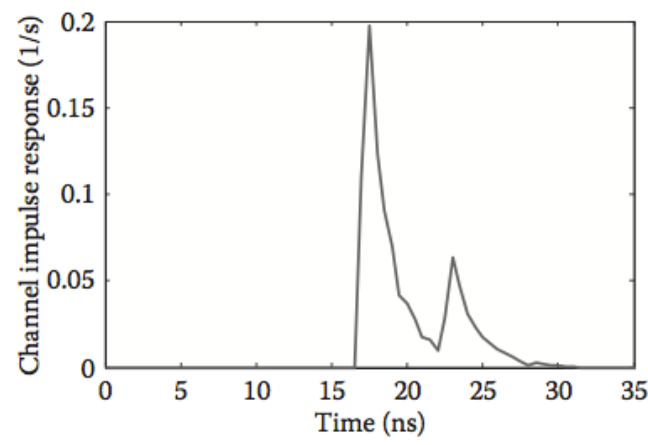

Fig. 5. Channel response $h(t ; S, R)$.

Regarding the power distribution, for a given power emitted by the LED bulb, in Fig. 6 the received power distribution is 
depicted. The predominance of LOS beam between transmitter and receiver is clearly observed.

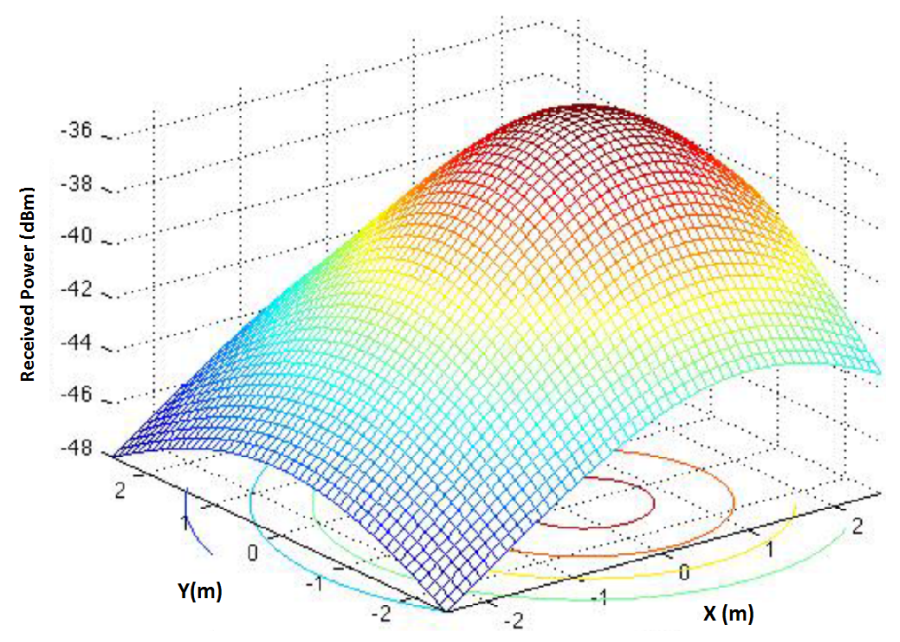

Fig. 6. The distribution of the received light power.

\section{PRototype Description}

The LiFi prototype system is based on an Software Defined Radio (SDR) approach. FPGAs boards have been used to implement the communication module in both the streetlight and the user equipment. The goal is to validate the communication function using this prototype in a scenario with streetlights. The prototype is divided into two subsystems: hardware and software subsystem. Fig. 7 shows the overall setup of the proposed testbed for the LiFi prototype.

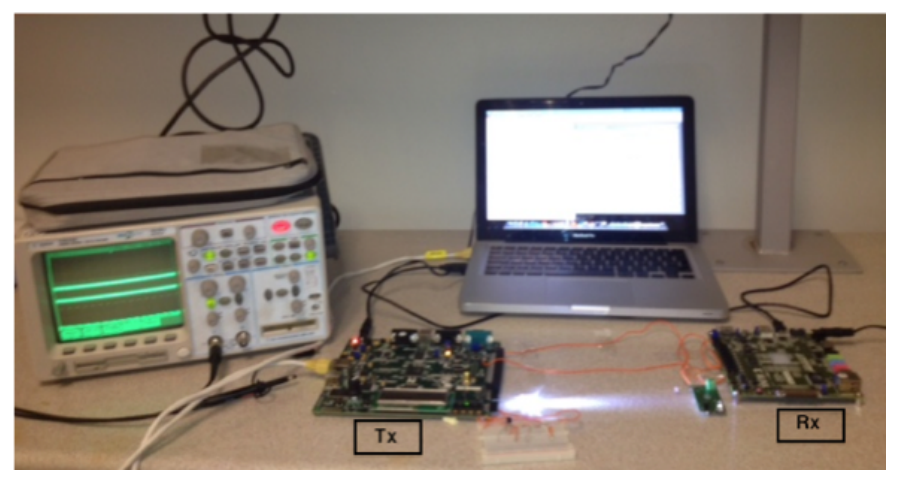

Fig. 7. Testbed for LiFi Prototype System.

\section{A. Hardware}

The transmitter and receiver designs have been implemented on two separate FPGAs. The FPGA used in the receiver has a larger memory, in order to store the performance measurements done at this end.

The transmitter front-end, the streetlight, is constituted by the Development Kit Board of Digilent, equipped with a Spartan 3E-xc3s500E FPGA, acting as the LiFi modulator. It also includes the LED driver circuit and a commercial high power white LED as the light emitting source. The data stream received from PLC is delivered via an Ethernet port to the FPGA and encoded as shown in Fig. 2, then the modulated signal is fed to the LED driver circuit, which acts in switching mode, varying the current of the streetlight LED.
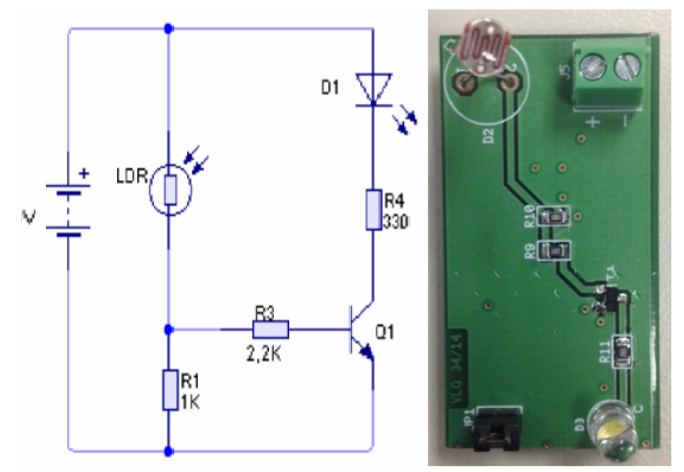

Fig. 8. Set up and prototype receiver.

The receiver front-end, at the phone's camera, consists of the Altys platform equipped with a Spartan 6-xc6slx16 FPGA. An external circuit, shown in Fig. 8, has been designed and implemented to capture the signal using an LDR (light-dependent resistor). The digital signal is delivered to demodulator FPGA to reverse the flow in Fig. 2. Also, the FPGA generates the clock signal in both Tx and Rx. The hardware description is depicted in Fig. 9

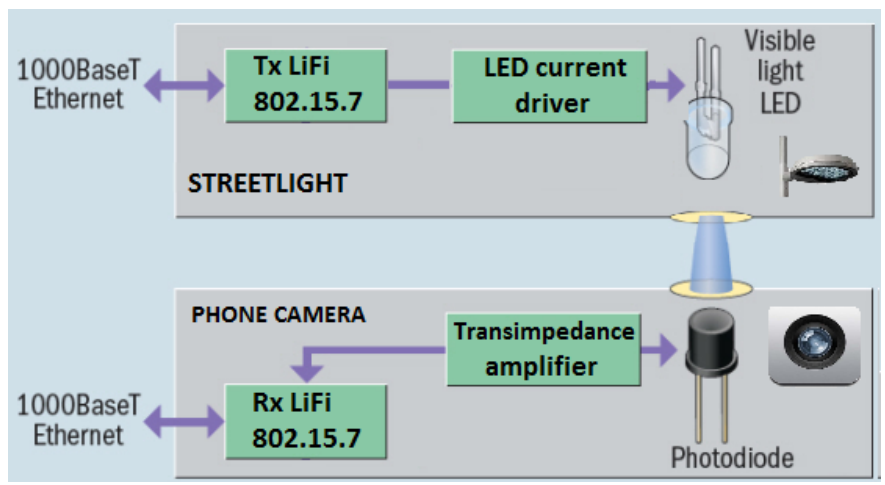

Fig. 9. Diagram of the LiFi Hardware Architecture.

\section{B. Software}

The software subsystem is responsible to implement the functions of each block represented in Fig. 2 in VHDL on FPGA. The Xilinx ISE Design Suite 14.7 tool is used. To simulate each implemented block, iSim Simulator integrated in Xilinx ISE tool and isused.

The data stream is delivered to FPGA, to that end, the Xilinx libraries are used to setup the LAN83C185 10/100/1000 ETH PHY port of the FPGA and to acquire the data to be encoded that allow us to use fewer FPGA resources. Also, memory blocks as buffers have been implemented using Xilinx libraries, in particular, to reduce the period of latency which is defined as the time is taken to deliver the first frame to encode to the RS block. 


\section{Measurements and Results}

The first phase of project has focused on the measurements to validate the transmitter and the channel. In the first experiment, the modulated signal is checked and compared to the simulations obtained with iSim simulator. Fig. 10 shows the OOK modulated signal using FPGA. Note that the frequency is $100 \mathrm{KHz}$ according to the standard. The initial latency, using the FPGA clock of $25 \mathrm{MHz}$, is $0.25 \mu \mathrm{s}$. Table IV summarizes the resource occupied by the modulator. RAM resources are used for the buffers.

TABLE IV

\begin{tabular}{|c|c|c|}
\hline \multirow{2}{*}{$\begin{array}{c}\text { FPGA } \\
\text { resources }\end{array}$} & \multicolumn{2}{|c|}{ Tx } \\
\cline { 2 - 3 } & Used & $\%$ \\
\hline \hline Slice LUTs & 4672 & 6 \\
\hline Slice Registers & 7306 & 8 \\
\hline Bonded IPBs & 104 & 11 \\
\hline Block RAMs & 16 & 13 \\
\hline
\end{tabular}

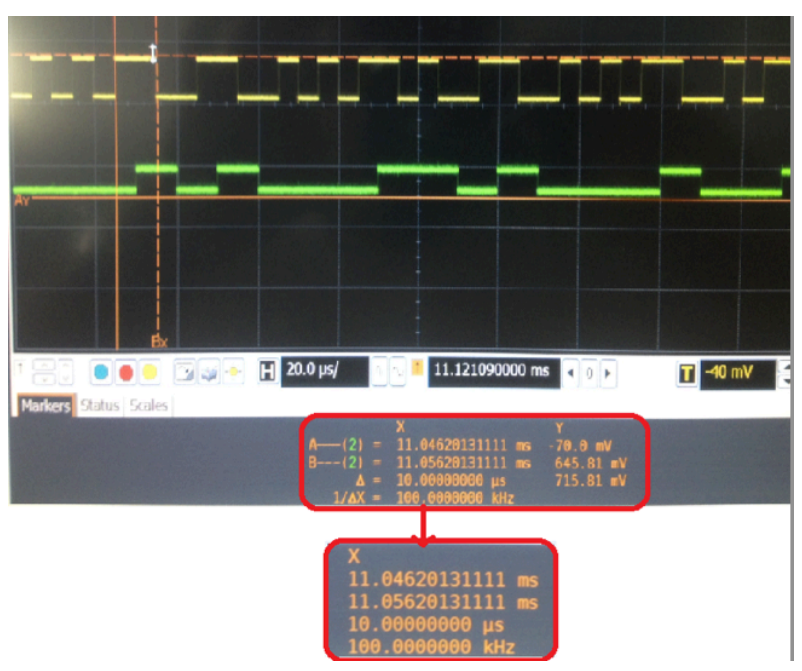

Fig. 10. Modulated real LiFi signal.

Secondly, the channel is measured. The measurements are taken with the transmitter and receiver being collinear with each other and varying the distance between them from $1 \mathrm{~cm}$ to $50 \mathrm{~cm}$ in $5 \mathrm{~cm}$ steps. Two cases are considered: in the first one, the ambient light is considered, while in the second case it is not taken into account. Fig. 11 shows the percent of current that is captured by the receiver. Although the ambient light influences in the quality of the received signal, LDR can detect the signal successfully. This maximum distance in the practice must be higher depending on the high of the streetlight. For this reason, when the LiFi is integrated in real scenario, amplifiers are needed.

\section{CONCLUSIONS AND ONGOING WORK}

In this paper, a LiFi system prototype implementing was shown. All the operating modes of the physical layer according to the specifications of the IEEE 802.15.7 standard are implemented. This development are based on FPGAs. Besides,

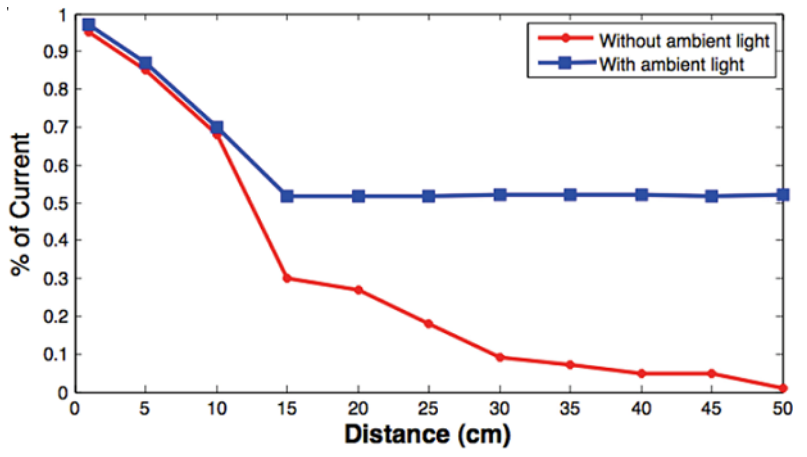

Fig. 11. Percent of captured current in the receiver.

the prototype includes a circuit, to capture the received signal, which has been design.

The testbed is proposed for a scenario with streetlights, for which the results have been validated. The transmitter and the channel have been tested. The effect of the ambient light has been observed.

Future works are focused on improving the achieved rates using more sophisticated modulation scheme such as OFDM. As well as, performing the same work with the receiver.

\section{ACKNOWLEDGEMENT}

This work has been partly funded by Ministerio de Industria, Energía y Turismo of Spain and the Company UVAXCONCEPTS, within Plan Nacional de Investigación Científica, desarrollo e inovación Tecnológica 2013-2016 with project TSI-100502-2013-024. The authors would like to thanks UVAX-Concept for funding and support.

\section{REFERENCES}

[1] D.V. Gibson, G. Kozmetsky, R.W: Smilor, "The technopolis Phenomenon: Smart Cities, Fast Systems, Global Networks,” Ed. Rowman and Littlefield, 1992.

[2] A. Zanella, N. Bui, A. Castellani, L. Vangelista, M. Zorzi, "Internet of Things for Smart Cities," IEEE Journal Internet of Things, vol. 1, pp. 22-32, Feb. 2014

[3] C. Doukas, F. Antonelli, "A full end-to-end as a service for smart city applications," IEEE 10th International Conference on Wireless and Mobile Computing, Networking and Communications (WiMob), pp. 181 186, 2014.

[4] J.G. Andrews, S. Buzzi, Wan Choi, S.V. Hanly, A. Lozano, A.C.K. Soong, J.C. Zhang, "What will 5G be?," IEEE J. Selected Areas in Commun., vol. 32, pp. 1065-1082, 2014.

[5] "Eurobarometer 73.3, Electromagnetic Fields. June 2010." [Online]. Available:

[6] J. Vucic and K.D. Langer, "High-Speed Visible Light Communications: State-of-art," in Optical Fiber Communication Conference (OFC'12), Los Angeles, USA, Mar. 2012.

[7] D. Tsonev, S. Videv and H. Haas, "Light Fidelity (Li-Fi): Towards AllOptical Networking," Proc. Broadband Access Communication Technologies VIII, vol. 9007, 2013.

[8] H. Haas, "Wireless data from every bulb," TED Global. Edinburg, Scotland 2011.

[9] IEEE, "Standard for Local and metropolitan area networks, Part 15.7: Short Range Wireless Optical Communication Using Visible Light," Rev. 802.15.7-2011, 2011.

[10] J.R. Barry, J. M. Kahn, W. J. Krause, E.A. Lee and D.G. Messerschmitt, "Simulation of multipath impulse response for indoor wireless optical channels," IEEE J. Select. Areas Commun., vol. 11, pp. 367-379,1993. 